\title{
BMJ Open Clinical course of pain and disability in patients with subacromial shoulder pain: a systematic review protocol
}

\author{
Zohreh Jafarian Tangrood, ${ }^{1}$ Angela Spontelli Gisselman, ${ }^{1,2}$ Gisela Sole, ${ }^{1}$ \\ Daniel Cury Ribeiro ${ }^{1}$
}

To cite: Tangrood ZJ, Gisselman AS, Sole G, et al. Clinical course of pain and disability in patients with subacromial shoulder pain: a systematic review protocol. BMJ Open 2018;8:e019393. doi:10.1136/ bmjopen-2017-019393

- Prepublication history and additional material for this paper are available online. To view these files, please visit the journal online (http://dx.doi. org/10.1136/bmjopen-2017019393).

Received 30 August 2017 Revised 14 March 2018 Accepted 3 April 2018

Check for updates

${ }^{1}$ School of Physiotherapy, Centre for Health, Activity, and Rehabilitation Research (CHARR), University of Otago, Dunedin, New Zealand

${ }^{2}$ Department of Physical Therapy, High Point University, High Point, North Carolina, USA

Correspondence to Dr Daniel Cury Ribeiro; daniel.ribeiro@otago.ac.nz

\section{ABSTRACT}

Introduction Previous systematic reviews have identified the multiple factors impacting on the prognosis of shoulder pain. However, the typical clinical course of subacromial shoulder pain remains unclear. The aim of this systematic review is to assess the clinical course of pain and disability in patients with subacromial shoulder pain.

Method and analysis We will systematically search electronic databases (Medline, Embase and AMED (via Ovid), Web of Science, Cochrane Library and Scopus) from the date of inception to 28 February 2018 for randomised controlled trials (RCTs) and observational studies with patients with subacromial shoulder pain. We will follow patient groups measured with pain and disabilities with no intervention or usual care treatment. Two reviewers will extract the data, and assess the risk of bias within included studies. We will use the Cochrane Risk of Bias tool for RCTs, and a methodological quality assessment tool for observational studies. We will perform metaanalyses for changes in pain and disability scores for each group (usual care and no intervention) at different time categories (up to 6 weeks, 6-12 weeks, 12-26 weeks or more). If there are 10 or more studies, we will perform a meta-regression for each planned analysis. The outcomes of the included studies will be described individually if the above statistical methods are not possible. Findings from this review will be of interest to clinicians and researchers, and will describe the expected clinical course of subacromial shoulder pain. This study may indicate the potential differences between the clinical courses of pain and disability over time.

Ethics and dissemination This review will not gather original data, hence ethical approval is not required. The results of this systematic review will be published in a peer-reviewed journal and presented at a scientific conference.

PROSPERO registration number CRD42016052518.

\section{INTRODUCTION}

Shoulder pain is the third most common musculoskeletal disorder. ${ }^{1}$ The annual prevalence of shoulder complaints, including trauma, frozen shoulder and subacromial shoulder pain, has been estimated to be $51 \%$. $^{2}$ Among shoulder complaints, subacromial shoulder pain is the most common

\section{Strengths and limitations of this study}

This study will be the first systematic review of observational and randomised controlled trials studies that followed patients with subacromial shoulder pain who received no intervention or usual care.

- This study may synthesise the outcomes of pain and disability of a large population of patients with subacromial shoulder pain at multiple time points.

- Studies included in this review may present diverse forms of 'usual care interventions' and clinical presentation (eg, duration of shoulder symptoms). Such heterogeneity may limit conclusions that can be drawn from this review.

- Depending on the number of articles included in this review, we may not be able to conduct meta-regression. This may limit the conclusions that can be drawn from this review.

disorder, ${ }^{3}$ representing $89 \%$ of total shoulder complaints referred to general practitioners (GPs) and physiotherapists. ${ }^{4}$ Subacromial shoulder pain is defined as pain at the shoulder joint radiating in the area between the neck and elbow that worsens with arm movements, especially during overhead activities. ${ }^{56}$ The annual prevalence of subacromial shoulder pain has been estimated as $7 \% .^{7}$

The clinical course of subacromial shoulder pain is currently unclear. Previous systematic reviews and studies described the stage of the disorder (eg, acute or chronic), ${ }^{8}$ personal (age) and shoulder pain disease characteristics (ie, the duration and amount of disability $)^{910}$ and the type of primary care treatment impact the clinical course recovery in patients with subacromial shoulder pain. $^{2} 11$ For example, $50 \%$ of patients presenting with acute subacromial shoulder pain may improve after 6 months, and $40 \%$ after 1 year. ${ }^{2}$ These findings suggest that $10 \%$ of patients with acute subacromial shoulder pain do not recover. In addition, $50 \%$ of patients with chronic subacromial shoulder pain recover after $10^{12}$ or 18 months of onset 
of symptoms. ${ }^{13}$ Observational studies estimating the clinical course of subacromial shoulder pain are either scarce, ${ }^{8}$ or have only presented the percentage of patients who have fully recovered. ${ }^{12} 14$ The wide range of recovery rates reported by previous studies suggests the clinical course for these patients is challenging and unclear.

Thus, the aim of this review will be to systematically assess the clinical course of pain and disability of patients with subacromial shoulder pain in usual care and no treatment conditions. The second aim will be to investigate whether pain and disability have similar clinical courses.

\section{METHODS}

\section{Search strategy}

We will search Medline, Embase and AMED (via Ovid), Web of Science, Cochrane Library and Scopus from the date of inception. The following search terms will be used for the studies' inclusion population: "Shoulder pain", "Shoulder impingement syndrome", "Shoulder problems", "rotator cuff", "Subacromial pain syndrome", "Subacromial impingement", "Painful arc", "Shoulder complaints", "Musculoskeletal disorders", "Subacromial injection", "shoulder disorders", "non-specific shoulder", "painful arc". Search terms to identify the type of intervention include: "No intervention", "no treatment", "standard care", "usual care", "waiting list", "wait and see", "wait-and-see", "no exercise", "ergonomics", "usual therapeutic approach". Terms we will use to identify prognosis include: prognosis, "clinical course", "natural course", inception, follow-up, recovery, "course of pain" and "course of shoulder". The completed search strategy used for all databases is summarised in online supplementary appendix 1. In addition, the reference lists of included studies for relevant studies will be searched.

\section{Study selection}

Two reviewers will screen the titles and abstracts of each citation retrieved from the electronic search (ZJT and DCR). Each citation will be selected based on the following eligibility criteria: (1) involved participants with shoulder pain; (2) published in all languages; (3) was a randomised controlled trial (RCT) and observational studies; (4) involved in usual care or no intervention; (5) we will include RCTs with $>2$ weeks of follow-up. We will exclude RCT studies if participants received any form of experimental intervention including placebo, sham or another preplanned specific treatment. Placebo and sham studies will be excluded as these interventions may have psychological effects that influence reported pain and disability scores. ${ }^{15} 16$

Two reviewers will obtain the full-text of potentially relevant articles and assess whether articles meet the inclusion criteria. In the case of disagreement, the third reviewer (ASG) will be consulted and final decision will be made by consensus. Studies that recruited patients with neck and shoulder pain will be included when the study reports shoulder pain characteristics (sample size and continuous data) separate from neck pain. In case of insufficient data, a reviewer will contact the authors and request additional information to assess whether the study meets the inclusion criteria.

\section{Participants}

We have defined subacromial shoulder pain as pain and discomfort in the shoulder joint with or without referred pain into the arm. ${ }^{5617}$ We will include studies with participants with subacromial shoulder pain or other diagnostic terms including subacromial pain disorders, rotator cuff tendinopathy, rotator cuff syndrome or disease, rotator cuff-related pain syndrome, rupture or tears of rotator cuff muscles, subacromial bursitis, anterolateral shoulder pain or non-specific shoulder pain. We will exclude articles with participants who have shoulder pain due to neurological disorders, painful hemiplegia, capsulitis (frozen shoulder), severe trauma, shoulder fracture, instabilities (dislocation), shoulder surgery or if patients have shoulder pain concomitant with systematic disease (eg, diabetes, cancer and rheumatoid disorders). We will exclude articles if $>5 \%$ of participants with reported shoulder pain are due to any of the conditions listed in the above exclusion criteria. ${ }^{18}$

\section{Type of intervention}

We will include observational studies and RCT studies that included groups of participants with subacromial shoulder pain receiving no intervention or usual care.

For the purpose of this review, we defined 'usual care' as any form of primary healthcare treatment routinely prescribed by GPs and physiotherapists for patients with subacromial shoulder pain. ${ }^{5819}$ Usual care might include the following: education, advice, oral analgesics, non-steroidal anti-inflammatory drugs, physiotherapy, corticosteroid injection, massage and exercise prescription, as long as the authors clearly stated that these were the part of usual care offered to patients in that respective healthcare system. ${ }^{5}$

\section{Outcomes}

We will extract mean and standard deviation (SD) scores for pain and disability over time.

\section{Data extraction}

For each eligible study, two reviewers (ZJT and ASG) will independently extract the following summary data: study design, country, sample size, participants' characteristics, symptom duration, group allocation (usual care and no intervention), follow-up time points and outcome measures (pain and disability). We will provide a summary of data in a table format. The authors may be contacted if reported data are not complete. We will wait for 1 month to receive an answer. If we do not receive a response from the authors then the study will be excluded.

\section{Strategy for data synthesis}

We will assess the course of pain and disability within no treatment and usual care groups. When possible, we 
will assess the course of pain and disability in acute and persistent shoulder pain independently. Categorising the chronicity of shoulder pain will be performed based on participants' mean or median shoulder pain duration at baseline. When mean and median are not available, a reviewer will, whenever possible, establish the chronicity of subacromial shoulder pain according to the inclusion criteria used by the studies. ${ }^{20}$ We will consider subacromial shoulder pain with duration $<3$ months as acute, ${ }^{8122}$ and $\geq 3$ months as persistent shoulder pain. ${ }^{8}$ In order to standardise the outcome measures in the studies, we will convert the dependent outcome (pain and disability) to a common 0-100 scale.

\section{Risk of bias within included studies}

Two reviewers will independently assess the risk of bias within included studies. Disagreements will be resolved by discussion and, if needed, through consulting with a third reviewer. The overall risk of bias for RCT and observational studies will be reported individually for each domain and will be considered when interpreting the data.

We will use the Cochrane Risk of Bias tool for assessing the risk of bias of included RCT studies. ${ }^{23}$ This tool has six domains (selection bias, performance bias, detection bias, attrition bias, reporting bias and other bias), and each domain is rated as low, high and unclear risk of bias. For the purpose of this review, performance risk of bias will be regarded as low risk if blinding of participants is impossible or difficult to be implemented. Blinding is often not possible in non-pharmacological health behaviour or exercise trials, ${ }^{24}$ therefore, categorising the performance bias in the non-pharmacological trials as high risk of bias does not necessarily imply that the trial was poorly designed. ${ }^{24}{ }^{25}$ With regard to attrition, unbalanced dropout rates between groups is ranked as 'high' risk of bias. ${ }^{26}$ For the purpose of this systematic review, we will extract pain and disability scores from participants allocated to 'no intervention' or 'usual care' groups between baseline and follow-up time points. Unbalanced dropouts are unlikely to influence our analyses. Hence, we will rate RCTs as having 'high' risk of attrition bias if the no intervention or usual care group present dropouts $>20 \%$ at the final follow-up. ${ }^{27}$

We will assess risk of bias within observational studies using a tool proposed by Altman. ${ }^{28}$ This tool was successfully used in a previous systematic review with a similar design to ours. ${ }^{20}$ This tool has two items related to sampling, two items related to follow-up and one item related to prognostic outcome. Each item is categorised with a 'yes' or 'no'.

\section{Data and statistical analysis}

We will present a narrative synthesis of findings from all included studies. We will categorise studies as 'usual care' or 'no intervention'. We will conduct meta-analysis followed by meta-regression if we identify a sufficient number of studies for each of these analyses. If meta-analysis or meta-regression is not possible, a narrative discussion based on findings from individual studies will be presented.

We will conduct meta-analyses within each time category for pain and disability if $<10$ studies are identified. Time may be categorised in three or more sections including 0-6 weeks, 6-12 weeks and 12-26 weeks or more (depending on the common follow-up time points in studies). Within each time category, we will pool the estimates of change in pain or disability related to baseline scores. We will use RevMan V.5.3 (Copenhagen: The Nordic Cochrane Centre, The Cochrane Collaboration, 2014) to conduct the meta-analysis. For each meta-analysis, we will first convert raw data (mean and SD) associated with pain and disability to $0-100$ score so that higher scores represent higher levels of pain and disability. We will then calculate the mean and standard error (SE) of the difference between baseline and follow-up at each time category (e.g between 6 and 12 months' time category). If studies do not report the mean and SE of the difference in pain or disability between baseline and follow-up time points, the mean and SE difference will be calculated through RevMan V.5.3. We will use generic inverse variance to calculate the mean and SE differences between baseline and follow-up for each outcome of interest (ie, pain and disability). Random effect model will be used to standardise the between-studies variance during meta-analyses, such as variance due to differences in participant's' clinical characteristics or sequence of treatment in usual care intervention. ${ }^{29}$ When conducting the meta-analysis, we will assess the heterogeneity in the usual care and no intervention groups using $\mathrm{I}^{2}$. As per the Cochrane Handbook, $\mathrm{I}^{2}<30 \%$ will be considered as not important heterogeneity; between $30 \%$ and $60 \%$ as moderate; $60 \%-90 \%$ as substantial and $90 \%-100 \%$ as considerable heterogeneity. ${ }^{26}$

A meta-regression will also be conducted using ' $R$ ' Statistical Software ${ }^{30}$ if we have a minimum of 10 studies for a specific outcome (ie, pain or disability scores), within a respective group (ie, 'usual care' or 'no intervention'). The association between time as independent variable and pain or disability scores as dependent variables will be assessed. In addition, we will assess whether pain and disability scores present similar clinical courses. We hypothesise that the clinical course of pain will improve faster over time when compared with disability. ${ }^{20}$

We will conduct a subgroup analysis if sufficient data are available. Subgroup analysis will be based on the following: different types of subacromial pain syndrome as per definition used by authors from included studies (eg, full rotator cuff tear, partial rotator cuff tear and subacromial bursitis) and duration of symptoms (acute: 0 up to 3 months and persistent: $>3$ months). It is possible that the studies reporting usual care will comprise diverse forms of interventions. If that is the case, such studies may be subgrouped based on the type of the intervention (eg, GPs or physiotherapy). 
In order to assess the bias in meta-analysis results, we will use funnel plots. ${ }^{26}$ This analysis will be performed if we have at least 10 studies included in the meta-analysis. ${ }^{31}$

\section{CONCLUSION}

To our knowledge, this will be the first systematic review to assess the clinical course of subacromial shoulder pain. This review will provide estimates of the course of subacromial shoulder pain and disability (acute and persistent) over time, for patients who have not received treatment or usual care. Such information can be of value for future studies comparing the effect of an intervention with expected recovery rates for patients who received usual care or no intervention. In addition, this study will inform whether pain and disability have the same clinical course over time. In the case of heterogeneity due to study characteristics (populations, outcome measures and follow-up time points) and biases, findings from this systematic review will provide possible directions for standardisation of participants, outcome measures and follow-up time points in future observational studies.

\section{Patient and public involvement}

Patients and/or the public were not involved with the development of this research project.

Contributors ZJT and DCR conceived of the project. ZJT was responsible for the designing of the review. DCR is the guarantor. DCR, GS and ASG contributed to design of the review. All authors revised and approved the protocol for the study. All authors revised manuscript for important content and approved the final version.

Funding ZJT is supported by the University of Otago Doctoral Scholarship.

Competing interests None declared.

Patient consent Not required.

Provenance and peer review Not commissioned; externally peer reviewed. Data sharing statement None.

Open Access This is an Open Access article distributed in accordance with the Creative Commons Attribution Non Commercial (CC BY-NC 4.0) license, which permits others to distribute, remix, adapt, build upon this work non-commercially, and license their derivative works on different terms, provided the original work is properly cited and the use is non-commercial. See: http://creativecommons.org/ licenses/by-nc/4.0/

(c) Article author(s) (or their employer(s) unless otherwise stated in the text of the article) 2018. All rights reserved. No commercial use is permitted unless otherwise expressly granted.

\section{REFERENCES}

1. Urwin M, Symmons D, Allison T, et al. Estimating the burden of musculoskeletal disorders in the community: the comparative prevalence of symptoms at different anatomical sites, and the relation to social deprivation. Ann Rheum Dis 1998;57:649-55.

2. van der Heijden GJMG. Shoulder disorders: a state-of-the-art review. Best Pract Res Clin Rheumatol 1999;13:287-309.

3. van der Windt DA, Koes BW, de Jong BA, et al. Shoulder disorders in general practice: incidence, patient characteristics, and management. Ann Rheum Dis 1995;54:959-64.

4. Virta L, Joranger P, Brox Jl, et al. Costs of shoulder pain and resource use in primary health care: a cost-of-illness study in Sweden. BMC Musculoskelet Disord 2012;13:1.

5. Bergman GJ, Winters JC, Groenier KH, et al. Manipulative therapy in addition to usual care for patients with shoulder complaints: results of physical examination outcomes in a randomized controlled trial. $J$ Manipulative Physiol Ther 2010;33:96-101.

6. Littlewood C, Bateman M, Brown K, et al. A self-managed single exercise programme versus usual physiotherapy treatment for rotator cuff tendinopathy: a randomised controlled trial (the SELF study). Clin Rehabil 2016;30:686-96.

7. Jacobsson L, Lindgärde F, Manthorpe R. The commonest rheumatic complaints of over six weeks' duration in a twelve-month period in a defined Swedish population. Prevalences and relationships. Scand J Rheumatol 1989;18:353-60.

8. Reilingh ML, Kuijpers T, Tanja-Harfterkamp AM, et al. Course and prognosis of shoulder symptoms in general practice. Rheumatology 2008;47:724-30.

9. Chester R, Shepstone L, Lewis JS, et al. Predicting response to physiotherapy treatment for musculoskeletal shoulder pain: protocol for a longitudinal cohort study. BMC Musculoskelet Disord 2013;14:192.

10. Kuijpers T, van der Windt DA, van der Heijden GJ, et al. Systematic review of prognostic cohort studies on shoulder disorders. Pain 2004;109:420-31.

11. Kooijman M, Swinkels I, van Dijk C, et al. Patients with shoulder syndromes in general and physiotherapy practice: an observational study. BMC Musculoskelet Disord 2013;14:128.

12. Bonde JP, Mikkelsen S, Andersen JH, et al. Prognosis of shoulder tendonitis in repetitive work: a follow up study in a cohort of Danish industrial and service workers. Occup Environ Med 2003;60:E8.

13. Croft P, Pope D, Silman A. The clinical course of shoulder pain: prospective cohort study in primary care. BMJ 1996;313:601-2.

14. Bodin J, Ha C, Petit A, et al. Natural course of rotator cuff syndrome in a French working population. Am J Ind Med 2014;57:683-94.

15. Li W, Zhang SX, Yang Q, et al. Effect of extracorporeal shock-wave therapy for treating patients with chronic rotator cuff tendonitis. Medicine 2017;96:e7940.

16. Bennell K, Wee E, Coburn S, et al. Efficacy of standardised manual therapy and home exercise programme for chronic rotator cuff disease: randomised placebo controlled trial. BMJ 2010;340:c2756.

17. Brownson P, Donaldson O, Fox M, et al. BESS/BOA Patient Care Pathways: Traumatic anterior shoulder instability. Shoulder Elbow 2015;7:214-26.

18. Lee TS, Kilbreath SL, Refshauge KM, et al. Prognosis of the upper limb following surgery and radiation for breast cancer. Breast Cancer Res Treat 2008;110:19-37.

19. Geraets JJ, Goossens ME, de Bruijn CP, et al. Cost-effectiveness of a graded exercise therapy program for patients with chronic shoulder complaints. Int J Technol Assess Health Care 2006;22:76-83.

20. da C Menezes Costa L, Maher CG, Hancock MJ, et al. The prognosis of acute and persistent low-back pain: a meta-analysis. CMAJ 2012;184:E613-24

21. Ottenheijm RP, Cals JW, Winkens B, et al. Ultrasound imaging to tailor the treatment of acute shoulder pain: a randomised controlled trial in general practice. BMJ Open 2016;6:e011048.

22. Masters S, O'Doherty L, Mitchell GK, et al. Acute shoulder pain in primary care - an observational study. Aust Fam Physician 2007;36:473-6.

23. Higgins JP, Altman DG, Gøtzsche PC, et al. The Cochrane Collaboration's tool for assessing risk of bias in randomised trials. BMJ 2011;343:d5928.

24. de Bruin M, McCambridge J, Prins JM. Reducing the risk of bias in health behaviour change trials: improving trial design, reporting or bias assessment criteria? A review and case study. Psychol Health 2015;30:8-34.

25. Sangelaji B, Smith CM, Paul L, et al. The effectiveness of behaviour change interventions to increase physical activity participation in people with multiple sclerosis: a systematic review and metaanalysis. Clin Rehabil 2016;30:559-76.

26. Higgins JP, Green S. Cochrane handbook for systematic reviews of interventions. New Jersey, USA: John Wiley \& Sons, 2011.

27. Fewtrell MS, Kennedy K, Singhal A, et al. How much loss to followup is acceptable in long-term randomised trials and prospective studies? Arch Dis Child 2008;93:458-61.

28. Altman DG. Systematic reviews of evaluations of prognostic variables. BMJ 2001;323:224-8.

29. Gough D, Oliver S, Thomas J. An introduction to systematic reviews. 2nd edn. Los Angeles: SAGE Publication Ltd, 2017.

30. R RCT. A language and environment for statistical computing. Secondary A language and environment for statistical computing. Vienna, Austria: R Foundation for Statistical Computing.

31. Lau J, loannidis JP, Terrin N, et al. The case of the misleading funnel plot. BMJ 2006;333:597-600. 